\title{
Physical, Laboratory, Radiographic, and Endoscopic Workup for Clostridium difficile Colitis
}

\author{
Samantha J. Baker, MD ${ }^{1}$ Daniel I. Chu, $\mathrm{MD}^{2}$ \\ ${ }^{1}$ Department of General Surgery, University of Alabama at \\ Birmingham, Birmingham, Alabama \\ 2 Department of Gastrointestinal Surgery, University of Alabama at \\ Birmingham, Birmingham, Alabama
}

Address for correspondence Daniel I. Chu, MD, Department of Gastrointestinal Surgery, University of Alabama at Birmingham, KB427, 1720 2nd Avenue South, UAB, Birmingham, AL 35294-0016 (e-mail: dchu@uab.edu).

Clin Colon Rectal Surg 2020;33:82-86.

Abstract
Keywords
- Clostridium difficile
- C. difficile
- pseudomembranous
colitis
- endoscopy

Clostridium (reclassified as "Clostridioides") difficile colitis is a common nosocomial infection associated with increased morbidity and mortality. Like many clinical encounters, a focused history and physical examination will help to guide initial management. Further laboratory testing will assist with diagnosis through stool studies, and blood tests, such as white blood cell counts and serum creatinine, can help to stratify patients into illness severity groups for treatment decisions. Radiographic evaluation can be helpful in patients with severe disease and concern for complicated colitis. Endoscopic evaluation should be carefully considered in patients with suspected mucosal injury secondary to infections and plays a role when an alternative diagnosis is suspected. Treatment options depend on the clinical presentation and can range from antibiotic therapy to emergent surgery to fecal transplantation for recurrent episodes. Care for these patients is often challenging, but through a systemic workup the appropriate treatment may be delivered.
Clostridium difficile $\mathrm{a}$ is a toxin forming, gram-positive, anaerobic bacillus bacteria that colonizes the gastrointestinal tract when the gut flora has been disturbed. ${ }^{1,2} \mathrm{C}$. difficile produces two toxins that make it particularly virulent. Toxin $\mathrm{A}$ is an exotoxin that binds to the brush border of the intestine disrupting the integrity of the intestinal lining ${ }^{3-5}$ and toxin $B$ is a cytotoxin that destroys cytoskeletal structure of the enterocytes leading to the pathologic findings of pseudomembranes. $^{6-8}$ The route of transmission for colonization is fecaloral, and $C$. difficile infectious colitis is classically associated with a recent course of antibiotic therapy. ${ }^{1,2}$

Certain patient populations are considered more high-risk for both initial and recurrent $C$. difficile colitis. ${ }^{9}$ Older age, longterm facility residence, recent hospitalizations, or prolonged lengths of stay, all predispose patients to increased risk. ${ }^{9-11}$ Other high-risk populations include patients undergoing immunosuppression therapy, ${ }^{12}$ chronic kidney disease, ${ }^{13}$ cirrhosis, ${ }^{14}$ use of gastric acid suppressive medications, ${ }^{13}$ or inflammatory bowel disease. ${ }^{15,16}$ Just one dose of antibiotics

\footnotetext{
a The genus name "Clostridium" was reclassified as "Clostridioides" in 2016.
}

can be the inciting factor for $C$. difficile colitis; risk of infection increases with prolonged use of antibiotics and use of multiple antibiotic therapies. ${ }^{9-13}$

C. difficile colitis can manifest in a spectrum of disease presentations from an asymptomatic carrier to fulminant colitis with toxic megacolon. ${ }^{1,13,17}$ When evaluating patients with suspected $C$. difficile infections, the patient history should focus on identifying offending medication(s), such as antibiotics, and high-risk patient features. ${ }^{13}$ Additionally, the history should focus on determining the severity of the disease and whether the presentation is an initial or a recurrent infection. ${ }^{17}$ The classification of disease severity is paramount to patient care because it will determine initial treatment. $^{17-19}$

In addition to the history, all patients, suspected of a C. difficile colitis infection, will require a focused abdominal exam, basic serum and stool laboratory testing, and potential radiographic evaluation; however, the indication(s) for further advanced testing depend on the clinical manifestations of the patient. ${ }^{1,17-19}$ For the remainder of this article, the authors will describe the physical examination findings,

Copyright (c) 2020 by Thieme Medical Publishers, Inc., 333 Seventh Avenue, New York, NY 10001, USA. Tel: +1(212) 760-0888.
DOI https://doi.org/ 10.1055/s-0039-3400474. ISSN 1531-0043. 
laboratory data, and the radiographic and endoscopic evaluation of patients with mild-to-moderate colitis, severe colitis, and fulminate colitis. These distinct classifications for disease severity are based on expert opinion; prospective scoring systems and predictive models are lacking. ${ }^{1}$

\section{Mild-to-Moderate Colitis}

Patients determined to have mild-to-moderate colitis, typically endorse diarrhea without signs of systemic infection and have less laboratory derangements. ${ }^{1,17}$ Risk factors for this group, as mentioned previously, include the following: (1) antibiotic use, (2) recent hospitalization, (3) prolonged length of stay, (4) chemotherapy/immunosuppression, and (5) multiple comorbidities. ${ }^{13,17}$ Patients with enteral access who receive enteral feeds are also at an increased risk. ${ }^{17}$

\section{Physical Examination}

Patients with mild-to-moderate colitis are hemodynamically normal and stable. These patients may be febrile and usually present with abdominal discomfort and cramping. Diarrhea containing mucus and occult blood can be present, but patients with mild-to-moderate colitis typically do not present with frank hematochezia or melena., ${ }^{13,17}$ Their abdominal exam may be relatively benign or demonstrate some diffuse tenderness to palpation. Patients with mild-to-moderate colitis should not exhibit signs of guarding, rebound tenderness, or peritonitis.

\section{Laboratory Data}

Laboratory data for mild-to-moderate colitis include a mildly elevated white blood cell (WBC) count of 15,000 cells/mL or less. Patients also do not exhibit evidence of end-organ dysfunction and have a normal serum creatinine or one less than $1.5 \mathrm{mg} / \mathrm{dL}{ }^{13,17}$ Patients will have positive stool tests for $C$. difficile bacteria or toxins. ${ }^{1,13,17}$

There are a variety of diagnostic tests available for C. difficile toxin testing in the stool and these should only be collected in patients with clinically significant diarrhea. ${ }^{20}$ All of these tests require liquid stool. For patients with suspected $C$. difficile with an ileus and low liquid stool formation, a rectal swab may be used. ${ }^{21-23}$ There is no role for testing of asymptomatic patients nor is there a role for repeat testing within the episode of infection to indicate a test for a cure. Patients may continue to test positive throughout treatment and recovery. ${ }^{20,23-26}$

The available types of diagnostic tests to be used alone or in combination for the detection of $C$. difficile infection include the following: nucleic acid amplification test (NAAT), enzyme immunoassay (EIA) for C. difficile glutamate dehydrogenase (GDH), EIA for $C$. difficile toxins $A$ and $B$, cell culture cytotoxicity assay, and selective anaerobic culture. $^{1,27-29}$ NAAT uses polymerase chain reaction (PCR) to detect gene specific to toxic strains of $C$. difficile. It is very sensitive and specific and results are available within an hour. ${ }^{27-29}$ However, there can be a false positive result with patients whose samples are delayed in processing or who have received empiric antibiotic treatment. ${ }^{29,30}$ NAAT can also lead to overtreatment and over diagnosis, as it only detects the toxin genes but does not test for active toxin production. ${ }^{31}$ Therefore, this test should only be ordered on patients where is it clinically indicated to avoid overtreatment and identification of asymptomatic carriers. 1,13,17,19,27 EIA testing for GDH and for toxins A and B are typically used in combination. EIA for $C$. difficile GDH has high sensitivity and the results are available in less than an hour. ${ }^{27-29}$ This assay tests for an enzyme present in both virulent and nonvirulent strains of $C$. difficile; therefore, its sensitivity and specificity are improved when used in combination with EIA for $C$. difficile toxins. ${ }^{27-29}$ EIA for $C$. difficile toxins A and B is specific to virulent strains but carries a high-false negative rate given the large amount of toxin needed for a positive test. $^{27-29,32}$ Because of these limitations and low sensitivity at approximately $75 \%$, it is used in combination with the EIA GDH testing. 1,13,17,27,28 Algorithms for testing will vary by site, but a helpful guide to use of EIA GHD and toxin testing is shown in - Fig. 1. ${ }^{33}$ NAAT testing is used when discordant results are obtained. ${ }^{28,33}$ Selective anaerobic culture and cell culture cytotoxicity assay are both very sensitive and specific; however, the disadvantage to this testing is the amount of time needed before results can be reported. ${ }^{28,29,34}$ These studies are useful in patients with suspected $C$. difficile colitis and ileus or in epidemiologic testing and studies of outbreaks. $^{34}$

\section{Radiographic and Endoscopic Evaluation}

In patients with a classic presentation of mild to moderate disease, positive stool tests, and/or improvement with empiric antibiotic treatment, additional radiographic evaluation is not warranted. ${ }^{35}$ If an abdominal radiograph or computed tomography (CT) scan is obtained in patients with these criteria, the findings would be generally benign and nonspecific, possibly showing signs of colonic inflammation. In patients with this disease presentation, endoscopic evaluation is not indicated. ${ }^{35}$

\section{Severe Colitis and Fulminate Colitis}

Patients with severe colitis exhibit multiple bouts of diarrhea with evidence of systemic illness and evidence of laboratory derangement/end organ dysfunction. Risk factors for severe colitis include those for mild-to-moderate colitis plus advanced age (>65 years), history of chronic obstructive pulmonary disease, history of renal failure, and infection with the hypervirulent strain B1/NAP1/027. ${ }^{13,17,36}$

Fulminate colitis is also known as severe, complicated colitis. Patients with fulminate colitis have multiple bouts of diarrhea with evidence of systemic infection, such as hypotension and shock, with concern for bowel distention, such as megacolon. ${ }^{13,17}$ Risk factors for fulminate colitis include those for mild-to-moderate colitis and severe colitis plus history of a recent abdominal operation, diagnosis of inflammatory bowel disease, or intravenous immunoglobulin therapy. 13,17,36 These patients may present with an ileus or toxic megacolon and therefore with signs and symptoms of obstruction instead of diarrhea. 


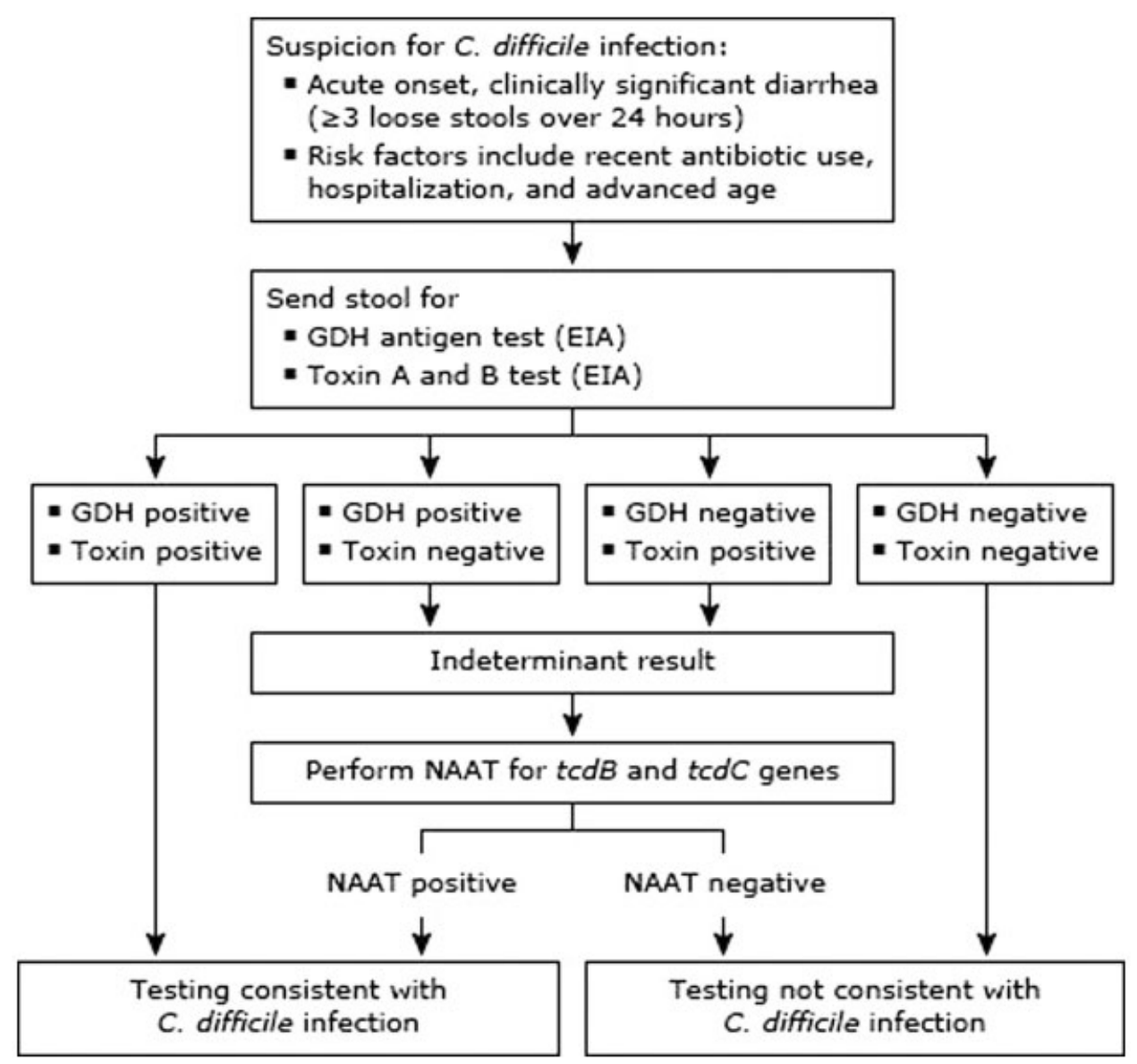

Fig. 1 UpToDate Algorithm for laboratory workup and diagnostic approach to C. difficile. ElA, enzyme immunoassay; GDH, glutamate dehydrogenase; NAAT, nucleic acid amplification test.

\section{Physical Examination}

Patients with severe colitis may be hemodynamically unstable and should be given antibiotics, supportive care, resuscitation, and consideration for admission to an intensive care setting. These patients are typically febrile and complain of diffuse abdominal pain, discomfort, and distention. Patient can also exhibit exam findings consistent with hypovolemia, such as dry mucus membranes and decreased skin turgor. ${ }^{1,13,17,19,36}$ Abdominal exam will reveal diffuse tenderness, distention, and high-pitched bowel sounds. Patients may have some voluntary guarding secondary to pain but should not have rebound tenderness or peritonitis.

It is important to mention that patients with acute C. difficile colitis may present with an ileus and therefore will not endorse the classic symptoms of watery diarrhea. ${ }^{1,36}$ In these patients, the diagnosis of $C$. difficile may be overlooked. Even if these patients present with a more benign exam, they can rapidly progress to severe colitis and even fulminate colitis with evidence of toxic megacolon or perforation. ${ }^{1,36}$

Patients with fulminant colitis are hemodynamically unstable with profound hypotension and shock. ${ }^{13}$ These patients require immediate administration of antibiotics and resuscitation in an intensive care setting. These patients are typically febrile and if not obtunded will complain of diffuse abdominal pain, discomfort, and distention. Abdom- inal exam will reveal diffuse tenderness, distention, and possibly diminished sounds, or a rigid abdomen. ${ }^{13,17}$ Patients may also exhibit guarding, rebound tenderness, and peritonitis. ${ }^{35}$ These exam findings should heighten the suspicion for perforation or impending perforation.

\section{Laboratory Data}

Laboratory data for severe colitis include WBC counts $>15,000$ cells $/ \mathrm{mL}$ and evidence of end organ dysfunction with creatinine levels $>1.5 \mathrm{mg} / \mathrm{dL}$ or greater than 1.5 -times with a baseline creatine. ${ }^{17}$ These patients can also have electrolyte derangements consistent with hypovolemia, evidence of lactic acidosis, and hypoalbuminemia. ${ }^{17,36}$ Patients will also have positive stool tests for $C$. difficile bacteria or toxins.

Laboratory data for fulminate colitis is similar to severe colitis with evidence of leukocytosis (or leukopenia) and an elevated serum creatine. ${ }^{17,36}$ These patients can also have electrolyte derangements consistent with hypovolemia and evidence of lactic acidosis. They will also exhibit evidence of systemic inflammation, such as hypoalbuminemia. ${ }^{36}$ Given the profound hypotension seen with fulminate colitis, there can also be evidence of end-organ dysfunction secondary to hypoperfusion, such as elevated liver enzymes. Patients will also have positive stool tests for $C$. difficile bacteria or toxins. 


\section{Radiographic and Endoscopic Evaluation}

In patients who are found to have severe disease manifestations, additional radiographic evaluation of the abdomen, and pelvis may be warranted. Additional imaging can be helpful to evaluate for small bowel or colonic dilation, bowel wall thickening, air/ fluid levels, or evidence of perforation. CT scan of the abdomen and pelvis with intravenous (IV) and by mouth ("per OS" PO) PO contrast is the ideal radiologic testing modality; plain films may be useful in circumstances when $\mathrm{CT}$ is unavailable. ${ }^{35}$ Plain abdominal X-rays may show dilated show dilated small and large bowel loops that are less than $7 \mathrm{~cm}$. CT scans can show some classic signs for colonic bowel wall inflammation and edema, such as "thumb printing" and "target signs." CT findings suggestive of pseudomembranous colitis include the accordion sign, which results from oral contrast trapped between edematous large bowel haustra with areas of bright contrast alternating with edematous bowel to resemble an accordion. ${ }^{35-39}$ Patients with fulminate colitis show evidence of free air indicating perforation or colonic distention indicating toxic megacolon which can be seen on either abdominal X-ray or CT scan. ${ }^{35}$ Toxic megacolon can be diagnosed based on radiographic evaluations showing dilated small bowel with air/fluid levels mimicking an obstruction or dilated colon up to $7 \mathrm{~cm}$ along in the setting of an infectious or inflammatory colitis. ${ }^{35}$

Endoscopic evaluation of patients with colitis is undertaken in certain circumstances when an alternative diagnosis is considered and/or there is the need for direct mucosal inspection and biopsy. Endoscopy can also be considered in patients with an ileus and suspected $C$. difficile infections, since stool tests will be less useful and visualization of the pseudomembranes will help to make the diagnosis. Patients with $C$. difficile exhibit these pseudomembranes on endoscopy (-Fig. 2) due to the toxins produced by the bacteria causing ulcerations in the mucosal surface. ${ }^{6}$ These are described as yellow/white plaques and their distribution can be continuous or patchy. ${ }^{6,35}$ Patients undergoing endoscopy would also undergo mucosal biopsy and C. difficile testing.

The absence of pseudomembranes does not rule out C. difficile colitis, and conversely, while pseudomembranes on endoscopy can help to diagnose patients with $C$. difficile colitis, they are not exclusive to that diagnosis. ${ }^{29}$ For example, several other infections and disease processes cause pseudomembranes in the setting of negative $C$. difficile testing, such as ischemic colitis, inflammatory bowel disease,


Fig. 2 Picture of pseudomembranous colitis on endoscopy. Arrows pointing at pseudomembranes. viral infections like cytomegalovirus, and parasitic infections with Entamoeba histolytica. ${ }^{29}$

If endoscopy is pursued, then limited flexible sigmoidoscopy should be considered to theoretically decrease the risk of perforation with little or no insufflation. Unfortunately, some patients may only show evidence of pseudomembranous colitis in the right colon or cecum area. ${ }^{35,40}$ However, for any case in which endoscopy is considered, one should proceed with caution. These evaluations should be performed at specialized centers under the care of experts. Patients who present with fulminate colitis and evidence of perforation or physical exam findings consistent with an acute abdomen warrant prompt surgical evaluation without the need for further endoscopic workup.

\section{Conclusion}

The treatment plan for patients with $C$. difficile colitis depends on the following factors: the severity of the disease at presentation, initial versus recurrent infection, and the overall clinical picture. ${ }^{13,17-19}$ The history, physical exam, and laboratory testing should all be used to aid in the workup and diagnosis of $C$. difficile colitis. Stool testing should follow protocols to ensure the highest specificity and sensitivity while remaining practical and time sensitive. Depending on patient presentation, radiographic and endoscopic testing can complement the workup to determine the most appropriate and effective treatment plan. Treatment options range from oral antibiotics therapy to urgent versus emergent surgery to consideration of fecal transplantation; these decisions are often made in combination with other medical professionals and services including surgery, critical care, gastroenterology, and infectious disease.

\section{Funding}

The authors do not have any financial disclosures or conflict of interest. This research was not supported by any funding agency.

\section{Conflict of Interest \\ None declared.}

\section{References}

1 McDonald LC, Gerding DN, Johnson S, et al. Clinical Practice Guidelines for Clostridium difficile Infection in Adults and Children: 2017 Update by the Infectious Diseases Society of America (IDSA) and Society for Healthcare Epidemiology of America (SHEA). Clin Infect Dis 2018;66(07):987-994

2 Bartlett JG, Moon N, Chang TW, Taylor N, Onderdonk AB. Role of Clostridium difficile in antibiotic-associated pseudomembranous colitis. Gastroenterology 1978;75(05):778-782

3 Price SB, Phelps CJ, Wilkins TD, Johnson JL. Cloning of the carbohydrate-binding portion of the toxin a gene of Clostridium difficile. Curr Microbiol 1987;16(01):55-60

4 Lima AA, Innes DJ Jr, Chadee K, Lyerly DM, Wilkins TD, Guerrant RL. Clostridium difficile toxin A. Interactions with mucus and early sequential histopathologic effects in rabbit small intestine. Lab Invest 1989;61(04):419-425

5 Sears CL, KaperJB. Enteric bacterial toxins: mechanisms of action and linkage to intestinal secretion. Microbiol Rev 1996;60(01):167-215 
6 Riegler M, Sedivy R, Pothoulakis C, et al. Clostridium difficile toxin $\mathrm{B}$ is more potent than toxin $\mathrm{A}$ in damaging human colonic epithelium in vitro. J Clin Invest 1995;95(05):2004-2011

7 Hecht G, Koutsouris A, Pothoulakis C, LaMont JT, Madara JL. Clostridium difficile toxin B disrupts the barrier function of T84 monolayers. Gastroenterology 1992;102(02):416-423

8 Alfa MJ, Kabani A, Lyerly D, et al. Characterization of a toxin Anegative, toxin B-positive strain of Clostridium difficile responsible for a nosocomial outbreak of Clostridium difficile-associated diarrhea. J Clin Microbiol 2000;38(07):2706-2714

9 Thomas C, Stevenson M, Riley TV. Antibiotics and hospital-acquired Clostridium difficile-associated diarrhoea: a systematic review. J Antimicrob Chemother 2003;51(06):1339-1350

10 Loo VG, Bourgault AM, Poirier L, et al. Host and pathogen factors for Clostridium difficile infection and colonization. N Engl J Med 2011;365(18):1693-1703

11 Kyne L, Sougioultzis S, McFarland LV, Kelly CP. Underlying disease severity as a major risk factor for nosocomial Clostridium difficile diarrhea. Infect Control Hosp Epidemiol 2002;23(11): 653-659

12 Kamthan AG, Bruckner HW, Hirschman SZ, Agus SG. Clostridium difficile diarrhea induced by cancer chemotherapy. Arch Intern Med 1992;152(08):1715-1717

13 Khanna S, Pardi DS. Clostridium difficile infection: new insights into management. Mayo Clin Proc 2012;87(11):1106-1117

14 Yan D, Chen Y, Lv T, et al. Clostridium difficile colonization and infection in patients with hepatic cirrhosis. J Med Microbiol 2017; 66(10):1483-1488

15 Rodemann JF, Dubberke ER, Reske KA, Seo DH, Stone CD. Incidence of Clostridium difficile infection in inflammatory bowel disease. Clin Gastroenterol Hepatol 2007;5(03):339-344

16 Singh H, Nugent Z, Yu BN, Lix LM, Targownik LE, Bernstein CN. Higher incidence of Clostridium difficile Infection among individuals with inflammatory bowel disease. Gastroenterology 2017;153(02): 430-438.e2

17 Bagdasarian N, Rao K, Malani PN. Diagnosis and treatment of Clostridium difficile in adults: a systematic review. JAMA 2015; 313(04):398-408

18 Drekonja DM, Butler M, MacDonald R, et al. Comparative effectiveness of Clostridium difficile treatments: a systematic review. Ann Intern Med 2011;155(12):839-847

19 Nelson RL, Suda KJ, Evans CT. Antibiotic treatment for Clostridium difficile-associated diarrhoea in adults. Cochrane Database Syst Rev 2017;3:CD004610

20 Gerding DN. Diagnosis of Clostridium difficile-associated disease: patient selection and test perfection. Am J Med 1996;100(05): 485-486

21 Kundrapu S, Sunkesula VC, Jury LA, Sethi AK, Donskey CJ. Utility of perirectal swab specimens for diagnosis of Clostridium difficile infection. Clin Infect Dis 2012;55(11):1527-1530

22 Peterson LR, Robicsek A. Does my patient have Clostridium difficile infection? Ann Intern Med 2009;151(03):176-179

23 Gerding DN, Johnson S, Peterson LR, Mulligan ME, Silva J Jr. Clostridium difficile-associated diarrhea and colitis. Infect Control Hosp Epidemiol 1995;16(08):459-477
24 Katz DA, Lynch ME, Littenberg B. Clinical prediction rules to optimize cytotoxin testing for Clostridium difficile in hospitalized patients with diarrhea. Am J Med 1996;100(05):487-495

25 Luo RF, Banaei N. Is repeat PCR needed for diagnosis of Clostridium difficile infection? J Clin Microbiol 2010;48(10):3738-3741

26 Sunenshine RH, McDonald LC. Clostridium difficile-associated disease: new challenges from an established pathogen. Cleve Clin J Med 2006;73(02):187-197

27 Crobach MJT, Baktash A, Duszenko N, Kuijper EJ. Diagnostic guidance for C. difficile infections. Adv Exp Med Biol 2018; 1050:27-44

28 Gateau C, Couturier J, Coia J, Barbut F. How to: diagnose infection caused by Clostridium difficile. Clin Microbiol Infect 2018;24(05): 463-468

29 Krutova M, Wilcox MH, Kuijper EJ. The pitfalls of laboratory diagnostics of Clostridium difficile infection. Clin Microbiol Infect 2018;24(07):682-683

30 Sunkesula VC, Kundrapu S, Muganda C, Sethi AK, Donskey CJ. Does empirical Clostridium difficile infection (CDI) therapy result in false-negative CDI diagnostic test results? Clin Infect Dis 2013;57 (04):494-500

31 Polage CR, Gyorke CE, Kennedy MA, et al. Overdiagnosis of Clostridium difficile Infection in the Molecular Test Era. JAMA Intern Med 2015;175(11):1792-1801

32 Bartlett JG. Clinical practice. Antibiotic-associated diarrhea. N Engl J Med 2002;346(05):334-339

33 Approach to Diagnosis of Clostridium difficile via EIA Screening followed by Reflex NAAT. UpToDate, Inc. https://www.uptodate. com/contents/clostridioides-formerly-clostridium-difficile-infection-in-adults-clinical-manifestations-and-diagnosis? search $=\mathrm{c}$.diff \%20testing\&source=search_result\&selectedTitle=1 150\&usage_type=default\&display_rank=1. Accessed October 31, 2019

34 Blossom DB, McDonald LC. The challenges posed by reemerging Clostridium difficile infection. Clin Infect Dis 2007;45(02): 222-227

35 Lamont JT, Kelly CP, Bakken JS. Clostridium difficile infections in adults: clinical manifestations and diagnosis. Available at: https://www.uptodate.com/contents/clostridium-difficile-infection-in-adults-clinical-manifestations-and-diagnosis?topicRef=2698\&source=see_link\# H7229445. Accessed October 31, 2019

36 Rubin MS, Bodenstein LE, Kent KC. Severe Clostridium difficile colitis. Dis Colon Rectum 1995;38(04):350-354

37 Kawamoto S, Horton KM, Fishman EK. Pseudomembranous colitis: spectrum of imaging findings with clinical and pathologic correlation. Radiographics 1999;19(04):887-897

38 Valiquette L, Pépin J, Do XV, et al. Prediction of complicated Clostridium difficile infection by pleural effusion and increased wall thickness on computed tomography. Clin Infect Dis 2009;49 (04):554-560

39 Lim J, Phillips AW, Thomson WL. An unexpected CT finding in a patient with abdominal pain. BMJ Case Rep 2013;2013;. Doi: 10.1136/bcr-2012-007345

40 Tedesco FJ. Antibiotic associated pseudomembranous colitis with negative proctosigmoidoscopy examination. Gastroenterology 1979;77(02):295-297 